\section{Topische Kortikoide bei allergischer Rhinitis} \section{Noch unter Wert gehandelt}

\author{
Moderne topische Glukokortikoide werden in Deutschland immer \\ noch vergleichsweise zurückhaltend eingesetzt. Dies dürfte sich \\ nun mit den jetzt verfügbaren modernen und sehr gut verträg- \\ lichen Substanzen wie Mometason ändern: Die systemische \\ Bioverfügbarkeit bei nasaler Anwendung ist zu vernachlässigen, \\ lokale Nebenwirkungen wie Nasenbluten bleiben aus. Sogar der \\ präventive Einsatz vor Beginn der Pollenflugsaison wird von den \\ Patienten als sehr positiv beurteilt.
}

$\mathrm{n}$ der Therapie der saisonalen allergischen Rhinitis spielen die topischen Glukokortikoide im Vergleich zu anderen Ländern eine eher unterbewertete Rolle. Ein Grund hierfür ist die immer noch bei Patient und Arzt herrschende "Kortisonangst" - entstanden in einer Zeit, als tatsächlich noch mit hohen Dosen viele unnötige Nebenwirkungen bei den Patienten induziert wurden.

\section{Keine Systemwirkungen}

Doch spätestens mit der Einführung von neuen Substanzen wie Mometason sollte die „Kortisonangst“ eigentlich endgültig besiegt sein. So besitzt Mometasonfuroat Nasenspray $\left(\right.$ Nasonex $\left.^{\circledR}\right)$ am Ort der Entzündung durch seine hohe Konzentration in der Nasenschleimhaut eine sehr gute Wirksamkeit, die systemische Bioverfügbarkeit liegt bei dieser Applikationsform aber unterhalb der Nachweisgrenze.

Diese günstigen Ergebnisse ließen eine Anwendung auch für Kinder als eine wirksame und sichere Therapieoption erscheinen. Studien konnten diese Überlegungen verifizieren, so dass die Substanz nun auch für Kinder ab 6 Jahren zugelassen ist (s. Kasten).

\section{Präventive Wirkung}

Die Vorteile einer frühzeitigen Intervention mit Glukokortikoiden sind in zahlreichen Untersuchungen zur allergischen Rhinitis klar herausgearbeitet worden, so R. Mösges (Köln). Dabei unterdrücken nasale Kortikoide nicht nur die allergischen Symptome des Heuschnupfens, sondern sie kontrollie- ren auch die pathophysiologisch entscheidende Entzündungsreaktion.

Da mittlerweile sehr gut verträgliche nasale Kortikoide zur Verfügung stehen, ging Mösges noch einen Schritt weiter: In einer multizentrischen Pilotstudie an 9 deutschen Kliniken mit rund 90 Patienten wurde die protektive Wirkung von Mometason u.a. auf die Symptomatik der saisonalen allergischen Rhinitis untersucht. Die Patienten wurden angewiesen, etwa 1 bis 2 Wochen vor Einsetzen „ihres“ Pollenflugs täglich einen Mometason-Sprühstoß pro Nasenloch vorzunehmen und dies während der Saison weiter zu führen.

\section{ECP-Werte bleiben normal}

„Man schafft mit dieser Dauermedikation in der Nase ein Mikroklima, unter dem alle relevanten Entzündungsparameter zurückgedrängt werden und es auch bei starker Pollenbelastung bei den Patienten zu keinen Rhinitissymptomen mehr kommen dürfte", so Mösges' Überlegungen.

Die Ergebnisse gaben der Theorie recht: Rund $80 \%$ der Studienteilnehmer äußerten sich sehr zufrieden mit der präventiven Gabe. Sie berichteten, dass es ihnen im Vergleich zu den Vorjahren unter einer Medikation z.B. mit H1-Blockern jetzt sehr viel besser gegangen sei. Manche haben sogar einen „Auslassversuch" gemacht, weil sie glaubten, sie hätten gar keine Rhinitis mehr.

Die ECP-Werte als wichtige Parameter einer Entzündungsreaktion lagen
Kinderzulassung für Nasonex ${ }^{\odot}$

Topische Kortikoide sind in der Behandlung von Kindern mit nicht mehr ganz leichten Formen der allergischen Rhinitis ein wichtiger Therapiebaustein, so U. Wahn (Berlin). Durch ihre antiinflammatorische Wirkung halten sie die Entzündungsreaktion in Schach und können so evtl. die Entwicklung eines Asthma bronchiale aufhalten.

Bei Kindern sollte auf eine besonders gute Verträglichkeit der Kortikoidtherapie geachtet werden. Dies ist mit neuen Kortikoiden wie Mometason möglich: Die Substanz hat bei nasaler Applikation keine nachweisbare systemische Bioverfügbarkeit, die Hypothalamus-Hypophysen-Nebennierenrinden-Achse wird nicht beeinflusst eine Wachstumsretardierung der Kinder ist damit nicht zu befürchten.

Dies hat auch eine Studie an 96 Kindern mit allergischer Rhinitis gezeigt, bei der unter einer täglichen Gabe von bis zu $200 \mu \mathrm{g}$ Mometasonfuroat über 14 Tage eine gute Wirksamkeit und Verträglichkeit festgestellt wurde.

Mometason ist deshalb nun auch für die Behandlung von Kindern mit einer allergischen Rhinitis ab 6 Jahren offiziell zugelassen.

\section{Wahn U}

Pressesymposium „Nasonex ${ }^{\circledR}$ - ein Jahr nach der Markteinführung“.

auch während der Pollenflugsaison im Bereich derer von Nicht-Allergikern, die allergische Früh- und Spätreaktion blieben aus. Nebenwirkungen wie $\mathrm{Na}$ senbluten wurden bei keinem Studienteilnehmer gesehen.

Die Compliance der Patienten in der Studie war überraschend hoch, nicht zuletzt wegen der einfachen Therapieanleitung: „Die Patienten wurden aufgefordert, das Kortikoidfläschchen neben ihr Zahnputzglas zu stellen und morgens nach dem Zähneputzen die Applikation vorzunehmen“, erklärte Mösges.

\section{Mösges $\mathbf{R}$}

Pressesymposium „Nasonex ${ }^{\circledR}$ - ein Jahr nach der Markteinführung “, veranstaltet von der Essex Pharma, Nürnberg, 2000. 